\title{
Subsidiarity and Weak Coupling in Wireless Networks
}

\author{
Aarne Mämmelä \\ Knowledge Intensive Products and Services \\ VTT Technical Research Centre of Finland, Ltd. \\ Oulu, Finland \\ Aarne.Mammela@vtt.fi
}

\author{
Jukka Riekki \\ Center for Ubiquitous Computing \\ University of Oulu \\ Oulu. Finland \\ Jukka.Riekki@oulu.fi
}

\begin{abstract}
We propose the subsidiarity and weak coupling principles for developing the sixth generation (6G) self-organizing wireless networks. The principles are common in social sciences and control theory, respectively. This proposal leads to organizing the network as a hierarchy of interacting rational agents with vertical and horizontal weak coupling. The central agent provides a performance goal and constraints to the lower level agents that operate almost autonomously in this multi-agent system. The system has various favorable properties, including stability, reliability, and efficiency. Present selforganizing networks are usually distributed without any centralized controller. The lack of a common externally given goal may lead to low performance, staggering behavior, or even chaotic situations. In communications, each transmitter can be interpreted as a rational lower level agent. A principle resembling subsidiarity, the locality principle, is used, for example, in cellular automata, systolic arrays, and edge computing. Subsidiarity is also a solution for the tragedy of the commons where common resources are overused because the costs are divided equally among the users, often with some significant delay. We also provide a historical review that shows each idea's origin because different disciplines use different terminology for similar concepts. Understanding the origins can reduce fragmentation and enhance scientific progress.
\end{abstract}

Keywords—hierarchy; modularity; subsidiarity; weakly coupled systems; autonomy; self-organizing communication networks; multi-agent systems; interacting agents; arbitrator; leader; tragedy of the commons

\section{INTRODUCTION}

New approaches are needed to implement the sixth generation $(6 \mathrm{G})$ self-organizing wireless networks. As these networks will have tight system requirements, they will be complex, but at the same time, they must be stable and efficient [1]. The requirements cannot be met by improving silicon

This article describes the work in the context of the DEDICAT 6G project under the European Union (EU) H2020 research and innovation programme (Grant Agreement No. 101016499). The contents of this publication are the sole responsibility of the authors and do not in any way reflect the views of the EU. This work was partly supported by the Academy of Finland 6Genesis grant 318927 . electronics' energy efficiency and miniaturization [2]. The reason is that we are close to the fundamental limits of nature, including the Szilard-Landauer limit and the Heisenberg limit [3]. Quantum computing provides an alternative, but presently only using either cryogenic temperatures or extremely high pressures. Hence, quantum computing is not energy efficient and cannot, at least in the near future, meet the energy efficiency requirements of mobile wireless communications.

We propose the use of subsidiarity and weak coupling principles to meet the requirements of self-organizing wireless networks. We selected self-organizing networks as the starting point due to the rich set of features they offer for wireless networks. Self-organizing systems are at the highest level in the hierarchy of technical systems and, therefore, the most complex and least mature [4]. They are usually distributed without any centralized control [5], [6]. However, welfare economics and game theory suggest that the optimum can only be found with strict conditions in a distributed system. In practice, some form of an arbitrator or leader is needed [7], [8]. Even in those ideal conditions, the distributed system tends to be unstable, and there is a trend towards inequity: a minority takes a majority of resources. This drift is called the Matthew principle [9]. Furthermore, the emergent macroscopic behavior is not easy to derive from microscopic behavior [10], and the global optimum is difficult to obtain with local interactions. Subsidiarity and weak coupling tackle these challenges.

Our main contribution is to present a multidisciplinary review on subsidiarity and weak coupling and apply it to selforganizing wireless networks. The review can help interested readers locate relevant research and apply the existing knowledge. The principles lead to organizing a system as a hierarchy of interacting rational agents Fig. 1). An agent is a feedback loop that consists of sensors, a decision block with a goal, and actuators that control the environment or process.

We are not aware of similar research. Generally, selforganizing systems are far from maturity [11]. In [12], the authors mention leaderless (decentralized or distributed) and leader-follow (centralized) multi-agent systems, but there is almost nothing about self-organization. In [13], the authors noticed that each mobile device is weakly coupled, but they did not further develop the idea.

Subsidiarity and weak coupling are common in social sciences and control theory, respectively. In social sciences, 


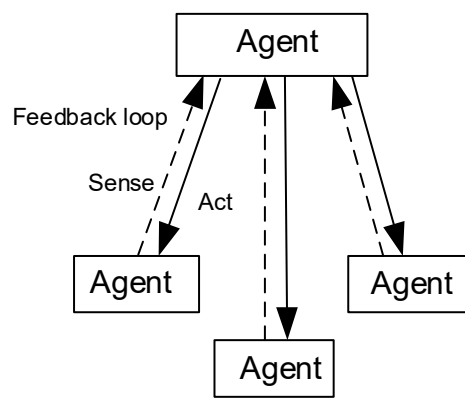

Fig. 1. A hierarchical multi-agent system using feedback loops.

subsidiarity is recognized as an efficient way to implement a hierarchical system. In control theory, the dynamics of many real physical systems are characterized by the presence of weak coupling among subsystems [13]. Subsidiarity is "the principle that a central authority should have a subsidiary function, performing only those tasks which cannot be performed at a more local level" [14]. Weak coupling may refer to coupling between hierarchy levels or between subsystems or agents at the same hierarchy level. Thus, subsidiarity is a more prescriptive principle since it clearly refers to a hierarchy of interacting agents where the lower level agents operate almost autonomously below the central agent. We believe that the principles will be widely useful in the 6th generation (6G) wireless networks. The actual optimization of the decision block is described in various general books, for example [15].

The rest of the paper is organized as follows. In Section II, we summarize the fragmented history of subsidiarity and weakly coupled systems, often called loosely coupled systems. In Section III, we present a transmitter as a rational agent. In Section IV, we show how a self-organizing system can be implemented with a set of interacting agents with weak coupling following the subsidiarity principle. In Section V, we draw some conclusions.

\section{HISTORY OF SUBSIDIARITY, WEAKLY COUPLED SYSTEMS, AND SELF-ORGANIZATION}

The history of subsidiarity, weakly coupled systems, and self-organizing systems is presented in Table I. A system consists of subsystems that are mutually coupled to form a whole. Thus the subsystems cannot be completely decoupled or noninteracting; otherwise, they would be separate systems. The degree of coupling may be weakly coupled, tightly coupled, or fully coupled [16], [17]. For example, in human-built digital systems, weak coupling can be realized by exchanging information through files or message passing, tight coupling using a common memory, and full or interleaved coupling with function calls. A cognitive radio system (1999) using sensing is an example of weak coupling, and federated learning (2017) is an example of tight coupling.

The coupling may be intentional in the form of information or unintentional in the form of interference. Thus, coupled subsystems are open systems that exchange matter, energy, or information with their environment. Information is not an independent quantity, but it is carried with matter or energy, such as in sound or radio waves. Communication is expensive in terms of energy, time, and bandwidth and hence should be minimized. The open system concept was introduced by Lotka (1925) [18]. Bertalanffy further developed the concept starting in 1932 [19].

TABLE I HISTORY OF SUBSIDIARITY, WEAK COUPLING, AND SELFORGANIZATION

\begin{tabular}{|l|l|}
\hline Year & \multicolumn{1}{|c|}{ Event } \\
\hline 300 s & Aristotle: subsidiarity \\
\hline 1826 & Gauss: RLS algorithm \\
\hline 1833 & Lloyd: tragedy of the commons \\
\hline 1925 & Lotka: open systems \\
\hline 1931 & Pius IX: subsidiarity \\
\hline 1947 & Ashby: self-organizing systems \\
\hline 1947 & Prigogine: start of complexity theory \\
\hline 1961 & Kleinrock: packet switching \\
\hline 1962 & Simon: hierarchy and modularity \\
\hline 1965 & Milne: weakly coupled systems \\
\hline 1967 & Falb and Wolovich: decoupling \\
\hline 1968 & Hardin: tragedy of the commons \\
\hline 1973 & Kahn: packet radio networks \\
\hline 1974 & Stevens: structured software design \\
\hline 1975 & Distributed artificial intelligence \\
\hline 1977 & Hewitt: intelligent agent \\
\hline 1984 & Widrow and Walach: orthogonalization \\
\hline 1993 & IEEE $802.11:$ ad hoc networks \\
\hline 1996 & Edge and cloud computing \\
\hline 1999 & Mitola: Cognitive radio systems \\
\hline 2017 & McMahan: Federated learning \\
\hline
\end{tabular}

In biology, self-organization is called morphogenesis [20]. Ashby (1947) developed the term self-organization [21]. The first self-organizing communication networks were based on packet switching invented by Kleinrock (1961) [22]. Arpanet, as proposed in 1967, was the first network based on packet switching [22]. The Arpanet was renamed the Internet in 1983. Kahn developed packet radio networks starting from 1973 [23]. After these first steps, self-organizing communication networks became popular in the 1980s [24]. The IEEE 802.11 subcommittee adopted the term ad hoc network in 1993, but the first ad hoc network was the packet radio network [25].

The subsidiarity principle is used in social sciences [26]. It leads to a hierarchical control system where the high level agents are weak. Most of the decisions are made locally by almost autonomous and almost isolated agents to avoid interference and delays that may create instability in the form of staggering behavior or even chaos. Subsidiarity targets 


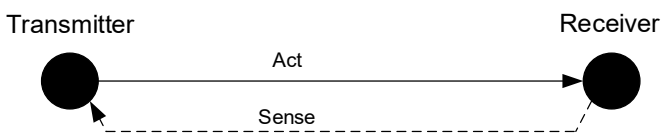

Fig. 2. Transmitter as a rational agent. The sensing information is provided by the receiver.

avoiding situations where everything depends on everything else. In general systems theory, the system separability principle tells us that system stability increases when the mean strength of interactions between subsystems decreases [27]. The principle became famous when Pope Pius IX selected it as one of the three main principles of the Catholic church in 1931. The US and EU constitutions are based on the subsidiarity principle. A principle resembling subsidiarity, the locality principle, is used, e.g., in cellular automata [28], systolic arrays [29], and edge computing.

The tragedy of the commons was first described by Lloyd in 1833 [30]. Later discussions by various authors, including Hardin (1968) [31], made the concept widely known. The tragedy of the commons surfaces when many actors use a common resource or commons, but the costs are divided equally, often with a significant delay, thus resulting in an overuse of the commons. The common resource was originally common land, but it can be any resource, for example, water or air. Hardin suggested solutions to the tragedy. Meadows summarized the solutions as educate and exhort, privatize the commons (decentralized solution), and regulate the commons (centralized solution) [31]. Applying the subsidiarity principle leads to a combination of privatization and regulation, as discussed in [32] with different terminology.

Simon (1962) applied near decomposability in physical, biological, and social systems [33]. His paper is usually considered the start of the theoretical work on the hierarchy concept [34]. Biological systems are nearly decomposable: they are hierarchical, modular, and weakly coupled both vertically from the whole system to subsystems and horizontally between subsystems. Milne (1965) published a paper on weakly coupled systems [35], which started a new research tradition resulting in a book in control theory [13]. Falb and Wolovic (1967) published a paper on the decoupling of multivariable control systems [36]. The original work by Simon, Milne, and Falb and Wolowich was done independently of each other. Therefore different authors often use different terminology.

Widrow and Walach (1984) proposed, also independently of the earlier authors, orthogonalized least-mean square (LMS) algorithm to solve a problem caused by strong coupling. Here, interference between the received samples from, for example, a radio channel creates coupling and correlation, forming a set of mutually coupled feedback loops that slow down the standard LMS algorithm [37]. The idea of the orthogonalized LMS algorithm is similar to the one used in the recursive leastsquares (RLS) algorithm that was invented by Gauss (1826) and Plackett (1950) [38]. Orthogonalization refers to decoupling. The term loose coupling has been used in administrative organizations [39].
Stevens, Myers, and Constantine (1974) proposed structured software design [40], originally developed by Constantine in the 1960s. Weakly coupled software modules improve reliability since an error cannot propagate easily. The idea is widely used in loosely coupled Internet services [41].

The idea of artificial intelligence was developed in 1956. Since the end of the 1970s, the focus has been on multi-agent systems [12], [42], [43]. A thermostat is an example of a simple agent. Sensors sense the state of the environment. The decision block makes decisions for the next actions to move the environment from the present state towards the desired goal state (goal-directed) or improved performance (maximization), usually iteratively using feedback [44], [45]. The actuators implement the decisions. The goal may include constraints. According to [45], the goal-directed and maximization principles are identical when there is only a single objective. The goal-directed principle works well in multiobjective optimization if the requirements are modest, but the maximization principle is a better approach. Other methods are described in [46]. Obviously, one does not often even know the desired state. In the maximization principle, some utility function is often used for scalarization.

Multi-agent systems consist of a set of interacting agents. Artificial intelligence is often defined as a theory of rational agents. Intelligence is based on deductive reasoning, but rationality is a more general concept defined as the ability to reach externally given goals successfully with limited resources [47]. Rationality is often called intelligence [44]. Rationality cannot be based on deduction only; thus, many alternatives are based on pattern recognition. Some hybrid systems use a combination of deduction or hard computing and pattern recognition or soft computing [16], [48].

In biology, complexity theory is essentially a theory of selforganization, and it can be implemented with a set of interacting agents [49]. The theory was initiated by Prigogine in 1947 using the open system concept [50]. McKelvey summarized the theory with seven first principles in an unpublished report in 2004 and published them in [49].

Communication networks should be efficient in terms of basic resources that we summarized in [4]. Research on network efficiency started from Milgram's (1967) small-world concept [51], [52]. The concept is useful in wireless networks [53] as small-world networks have high global and local efficiencies [54]. Network efficiency emphasizes the importance of the small-world concept in communication networks [52]. Even the human brain forms a small world [55].

\section{TRANSMITTER AS A RATIONAL AGENT}

In this section, the principles of the lower level agents in Fig. 1 are presented. The larger system, including the central agent, is discussed in the next section. A transmitter can be interpreted as a rational agent (Fig. 2). The transmitter receives part of the sensing information from the corresponding receiver through a feedback channel, usually in a reduced form. The agent makes decisions using the state of the channel estimated in the receiver. If, e.g., the receiver observes a frequency shift, it is corrected in the transmitter. The actuator transmits the signal in space, frequency, and time, and also selects the carrier 
phase. In the space domain, the actuator uses beamforming. In the amplitude, frequency, and time domains, it uses power, frequency, and timing control, respectively. Phase can be challenging to control in mobile communications. The phase can change rapidly due to multipath fading, and the loop delays may be considerable. When the transmitter and receiver are stationary, carrier phase control is a feasible approach.

The transmitter agent's primary aim is to avoid interference between different users when transmitting signals. Interference avoidance is possible if the signals are orthogonal [56]. The channel may attenuate, distort, and shift the transmitted signal in space, frequency, time, and carrier phase. Two signals are orthogonal if they are not overlapping in space, frequency, or time. Orthogonality is generally defined by using the signal space concept [57], [58]. The signals may be either coherently or noncoherently orthogonal. Coherently orthogonal signals are orthogonal only for a given phase shift. Noncoherently orthogonal signals are orthogonal with any phase shift, possibly generated by the channel.

\section{SELF-ORGANIZATION WITH INTERACTING AGENTS}

We can draw several conclusions from the historical review in Section II. Similar ideas have appeared independently many times with different terminology, and the literature tends to be disconnected. We can really understand the historical progress and state of the art and offer a vision to the future only when we know the origin of each concept and term. Complex systems are invariably composed of large numbers of rational agents, as shown in [59]. To reduce complexity, the systems must be hierarchical [34]. Thus a hierarchical multi-agent system is a natural choice to implement self-organization [11], [12], [49]. Subsidiarity with its weak horizontal coupling is known to be an efficient way to implement hierarchy, especially in social sciences [60]. Local decisions are preferred to avoid significant delays and possible instability. Since the agents are based on feedback loops that may be open to interference from the environment, we prefer weak horizontal coupling systems to avoid chaotic situations. The strongest reason to consider subsidiarity and weak coupling is that they are used in biological systems that are known to be efficient. In fact, evolution would not have had enough time unless these principles would not have been used [33]. In engineering, the weakly coupled systems have been used in structured software design [40], Internet services [41], and control theory [13]. Modern network architectures such as Experiential Networked Intelligence (ENI) are based on feedback [61].

The hierarchy of technical systems includes automatic and autonomous systems [4]. Automatic systems or automata do not need any manual control but may require some external control signals, i.e., the automata may be controllable by an outside agent [45]. Automatic systems include control and adaptive systems [4]. An external control signal or goal can be given in the form of a set-point value or a reference signal, also called a training signal. A control system almost always needs a set-point value. In a thermostat, it is the desired temperature.

Autonomous systems are automatic systems that do not need any external control, i.e., they are self-controlling. They still usually need a goal given by a human actor in the form of the desired state or performance to maximize [45]. The goal can only be provided by a human agent with free will, as no machine has such volition. Self-organizing systems are autonomous systems that can change their organization or structure. Most self-organizing systems developed so far have been distributed without centralized control, although the term self-organization only implies autonomy and does not exclude the use of centralized control. Lack of centralized control may lead to loss of optimality and even instability, which, in turn, can result in chaotic behavior. An externally given goal is one crucial way to obtain stability.

Self-organization can be formed using a hierarchy of interacting agents (Fig. 3) [49]. In our example of a transmitter and a receiver, the central agent coordinates the lower level agents shown in Fig. 2. Fig. 3 includes two wireless links and their mutual interference. The central agent offers goals and constraints to the lower level agents to ration the use of the basic resources and guarantees equity. Such a network is selforganizing since it can, e.g., route the signals through different lower level agents. The essential elements in self-organizing systems include hierarchy to reduce complexity and the degree of centralization to manage geographically distributed systems. Complexity can be reduced with different speeds as well as ranges and resolutions in amplitude, time, frequency, and space at different levels of hierarchy. At the lower levels, the speed is high, the range is restricted, and the resolution is high, and at the higher layers, the speed is slow, the range is broad, and the resolution is low [44], [62], [63], [64].

The three commonly known degrees of centralization are centralized control, decentralized control, and their intermediate form, distributed control [4]. In decentralized control, the agents are autonomous. In distributed control, the agents may cooperate at least with their nearest neighbors. Self-organizing systems are commonly based on distributed control. The subsidiarity principle determines another intermediate form used in social sciences. Subsidiarity is flexible because it offers all the three other degrees of centralization depending on the strength of the couplings.

A problem involving mutually conflicting objectives must be solved using multiobjective optimization, also called joint optimization [15], [65]. Such objectives are usually related to resource efficiency. An example is energy efficiency (in bit/J). The desirable optimum is generally called the Pareto optimum, a useful concept when the objectives are mutually commensurate. With many objectives, the optimum is not

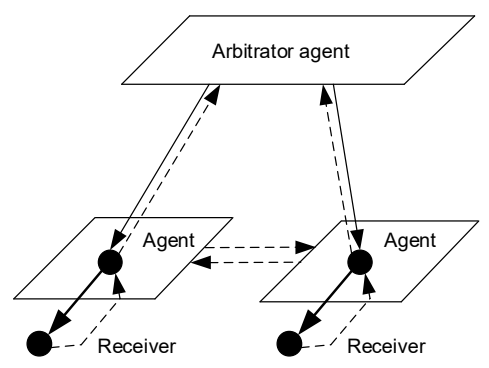

Fig. 3. Self-organizing communication network that is vertically and horizontally weakly coupled. 
unique but a set of optima, which are in general not equitable. It is well known from welfare economics [7] and game theory [8] that a system based on autonomous agents or players tends to drift towards undesirable and often suboptimal states which do not offer equity. Furthermore, different basic resources are incommensurate, and therefore there is no other way to solve the problem than to use an evolutionary approach using the law of supply and demand [9].

The Pareto optimum is challenging to find. Optimization problems, in general, have exponential complexity with respect to the size of the problem and therefore are mathematically intractable, and some heuristic methods must be used [15]. Welfare economics shows that a free market based on privatization [31] provides Pareto optimal solutions only in strict conditions that are in general not valid [7]. For example, the market must be perfectly competitive, and all the market participants must have perfect information. Simon's bounded rationality principle explains this: the subsystems generally have limited knowledge of the overall situation [31]. In the game theory, a game tends to converge to a Nash equilibrium, which is not in general Pareto optimal [8]. In fact, a game suffers from the Matthew principle where some monopolies appear. Equity is not a scientific but ethical question, which does not mean that it would be less important. If the players cooperate, they work as a single player, and the Pareto optimum can be found [65]. The use of an arbitrator can improve the situation in an ordinary game. The arbitrator is a central agent that can send private or public signals to the players, thus coordinating them. Such arbitration naturally leads us to the subsidiarity principle. An arbitrator may also be formed by several human actors that make agreements about regulation or rationing. A good example is the International Telecommunication Union - Radiocommunication Sector (ITU-R) that regulates radio frequencies and satellite orbits. We may conclude that subsidiarity has a good theoretical basis in many disciplines.

\section{CONCLUSION}

We have proposed subsidiarity and weak coupling for future self-organizing and other autonomous networks such as $6 \mathrm{G}$ networks to improve the optimality of these systems and solve the tragedy of the commons. The locality principle resembles subsidiarity. Since different disciplines are using different terminology for similar concepts, we provided a historical review that shows each idea's origin. Understanding the origins can reduce the fragmentation of science and enhance faster development. The subsidiarity principle exists in various forms in many disciplines but is not used in self-organizing communication networks, which are usually distributed networks. A system applying these principles consists of a hierarchy of interacting agents that are weakly or loosely coupled. The lower-level agents operate almost autonomously (vertical weak coupling) and have only minor interaction with each other (horizontal weak coupling). In communications, transmitters are agents. Weak centralized control is needed to provide a common goal, constraints, and equity with minimal control. Systems based on subsidiarity have many positive properties, including stability, reliability, and efficiency, thus reducing complexity. Stability is improved because of weak interactions between various agents and small delays due to mostly local decisions. Scalability is not as good as in decentralized and distributed systems, but this problem can be solved using hierarchy and negotiating agents. The system can converge fast to an optimal solution. Subsidiarity and weak coupling provide a good framework for optimization though the actual optimization and related protocols were out of this paper's scope. The selection of the performance goals and related constraints is an important problem for the future. Results from various disciplines are available, including multiagent systems, control theory, and robotics. Modularity combined with weak coupling produces a flexible, comprehensible, and reliable system since errors cannot easily propagate. The network must also follow the well-known small-world principles to obtain high network efficiency in addition to high resource efficiency. The problem with the incommensurability of the basic resources can be solved only with an evolutionary method using the law of supply and demand.

\section{ACKNOWLEDGMENT}

Discussions with Jyrki Huusko and Adrian Kotelba are gratefully acknowledged.

\section{REFERENCES}

[1] J. Riekki and A. Mämmelä, "Research and education towards smart and sustainable world," IEEE Access, vol. 9, pp. 53156-53177, 2021.

[2] T. N. Theis and H.-S. P. Wong, "The end of Moore's law: A new beginning for information technology," Computing in Science \& Engineering, 2017, vol. 19, no. 2, pp. 41 - 50, Mar.-Apr. 2017.

[3] A. Mämmelä and A. Anttonen, "Why will computing power need particular attention in future wireless devices?" IEEE Circuits and Systems Magazine, vol. 17, pp. 12-26, First Quarter 2017.

[4] A. Mämmelä, J. Riekki, A. Kotelba, and A. Anttonen, "Multidisciplinary and historical perspectives for developing intelligent and resourceefficient systems," IEEE Access, vol. 6, pp. 17464-17499, 2018.

[5] C. Prehofer and C. Bettstetter, "Self-organization in communication networks: Principles and design paradigms," IEEE Commun. Mag., vol. 43, no. 7, pp. 78-85, Jul. 2005.

[6] F. Dressler, "A study of self-organization mechanisms in ad hoc and sensor networks," Comput. Commun., vol. 31, no. 13, pp. 3018-3029, 2008.

[7] J. E. Stiglitz, "The contributions of the economics of information to twentieth century economics," The Quarterly Journal of Economics, vol. 115, no. 4, pp. 1441-1478, Nov. 2000.

[8] S. Lasaulce and H. Tembine, Game Theory and Learning for Wireless Networks: Fundamentals and Applications. Oxford, UK: Academic Press, 2011.

[9] K. E. Boulding, The World as a Total System. Beverly Hills, CA: SAGE, 1985.

[10] H. Silk, M. Homer, and T. Gross, "Design of self-organizing networks: Creating specified degree distributions," IEEE Transactions on Network Science and Engineering, vol. 3, no. 3, pp. 147-158, July-September 2016.

[11] D. Ye, M. Zhang, and A. V. Vasilakos, "A survey of self-organization mechanisms in multi-agent systems," IEEE Transactions on Systems, Man, and Cybernetics: Systems, vol. 47, pp. 441-461, Mar. 2017.

[12] A. Dorri, S. S. Kanhere, and R. Jurdak, "Multi-agent systems: A survey," IEEE Access, vol. 6, pp. 28573 - 28593, 2018. 
[13] Z. Gajic, M.-T. Lim, D. Skataric, W.-C. Su, and V. Kecman, Optimal Control: Weakly Coupled Systems and Applications. Boca Raton, FL: CRC Press, 2008.

[14] Oxford Learner's Dictionaries. [Online]. Available: https://www.oxfordlearnersdictionaries.com.

[15] E. G. Talbi, Metaheuristics: From Design to Implementation. Hoboken, NJ: Wiley, 2009.

[16] K. McGarry, S. Wermter, and J. MacIntyre, "Hybrid neural systems: From simple coupling to fully integrated neural networks," Neural Computing Surveys, vol. 2, no. 1, pp. 62-93, 1999.

[17] K. S. Decker, "Distributed problem-solving techniques: A survey," IEEE Trans. Syst., Man, and Cybern., vol. SMC-17, no. 5, pp. 729-740, Sep./Oct. 1987.

[18] D. Hammond, The Science of Synthesis: Exploring the Social Implications of General Systems Theory. Boulder, CO: University Press of Colorado, 2003

[19] L. von Bertalanffy, General System Theory: Foundations, Development, Applications, rev. ed. New York: George Braziller, 1971

[20] J. Briscoe and A. Kicheva, "The physics of development 100 years after D'Arcy Thompson's 'On Growth and Form'," Mechanisms of Development, vol. 145, pp. 26-31, 2017.

[21] S. G. Tzafestas, Energy, Feedback, Adaptation, and Self-Organization: The Fundamental Elements of Life and Society. Cham, Switzerland: Springer International Publishing, 2018.

[22] A. Huurdeman, The Worldwide History of Telecommunications. Hoboken, NJ, USA: Wiley, 2003.

[23] R. E. Kahn, S. A. Gronemeyer, J. Burchfiel, and R. C. Kunzelman, "Advances in packet radio technology," Proc. IEEE, vol. 66, no. 11, pp. 1468-1496, Nov. 1978.

[24] T. Robertazzi and P. Sarachik, "Self-organizing communication networks," IEEE Commun. Mag., vol. 24, no. 1, pp. 28-33, Jan. 1986

[25] R. Ramanathan and J. Redi, "A brief overview of ad hoc networks: Challenges and directions," IEEE Commun. Mag., vol. 40, no. 5, pp. 20 22, May 2002.

[26] M. Evans and A. Zimmermann (Eds.), Global Perspectives on Subsidiarity, Dordrecht: Springer, 2014,

[27] L. Skyttner, General Systems Theory: Problems, Perspectives, Practice, 2nd ed. Singapore: World Scientific Publishing, 2005.

[28] P. Sarkat, "A brief history of cellular automata," ACM Computing Surveys, vol. 32, no. 1, pp. 80-107, March 2000.

[29] S.-Y. Kung, "On supercomputing with systolic/wavefront array processors," Proc. IEEE, vol. 72, pp. 867-884, July 1984.

[30] D. Feeny, F. Berkes, B. J. McCay, and J. M. Acheson, "The tragedy of the commons: Twenty-two years later," Human Ecology, vol. 18, no. 1, pp. 1-9, March 1990.

[31] D. H. Meadows and D. Wright (Ed.), Thinking in Systems: A Primer. White River Junction, VT: Chelsea Green Publishing, 2008.

[32] K. E. Boulding, Three Faces of Power. Beverly Hills, CA: SAGE, 1989.

[33] H. A. Simon, "The architecture of complexity," Proceedings of the American Philosophical Society, vol. 106, pp. 467-482, Dec. 1962.

[34] J. Wu, "Hierarchy theory," in Linking Ecology and Ethics for a Changing World: Values, Philosophy, and Action, R. Rozzi, S. T. A Pickett, C. Palmer, J. J. Armesto, and J. B. Callicott, Eds., Springer, 2013, ch. 24, pp. 281-302.

[35] R. D. Milne, "The analysis of weakly coupled dynamical systems," Int. J. Contr., vol. 2, pp, 171-199, Aug, 1965.

[36] P. Falb and W. Wolovich, "Decoupling in the design and synthesis of multivariable control systems," IEEE Transactions on Automatic Control, vol. 12, no. 6, pp. 651-659, Dec. 1967.

[37] B. Widrow and E. Walach, "On the statistical efficiency of the LMS algorithm with nonstationary inputs," IEEE Trans. Inf. Theory, vol. IT30, no. 2, pp. 211-221, March 1984.

[38] P. C. Young, "Gauss, Kalman and advances in recursive parameter estimation," Journal of Forecasting, vol. 30, pp. 104-146, 2011.
[39] R. B. Glassman, "Persistence and loose coupling in living systems," Behavioral Science, vol. 18, pp. 83-98, 1973.

[40] W. P. Stevens, G. J. Myers, and L. L. Constantine, "Structured design," IBM Systems Journal, vol. 13, no. 2, pp. 115-139, 1974.

[41] C. Pautasso and E. Wilde, "Why is the web loosely coupled? A multifaceted metric for service design," in Proc. WWW'09.

[42] Y. Rizk, M. Awad, and E. W. Tunstel, "Decision making in multi-agent systems: A survey," IEEE Transactions on Cognitive and Developmental Systems, vol. 10, no. 3, pp. 514-529, Sep. 2018.

[43] Y. Demazeau and J. P. Muller (Eds.), Decentralized A.I. Amsterdam, The Netherlands: North Holland, 1990.

[44] J. S. Albus and A. M. Meystel, Engineering of Mind: An Introduction to the Science of Intelligent Systems. New York: John Wiley \& Sons, 2001.

[45] D. McFarland and T. Böser, Intelligent Behavior in Animals and Robots. Cambridge, MA: MIT Press 1993.

[46] J. C. Bezdek, "(Computational) intelligence: What's in a name?" IEEE Systems, Man \& Cybernetics Magazine, vol. 2, no. 2, pp. 4-14, April 2016.

[47] D. Robson, The Intelligence Trap: Why Smart People Do Stupid Things and How to Make Wiser Decisions. London: W. W. Norton \& Company, 2019.

[48] L. R. Medsker, Hybrid Intelligent Systems. New York: Springer, 1995.

[49] H. Benbya and B. McKelvey, "Toward a complexity theory of information systems development," Information Technology \& People, vol. 19 , no. 1, pp. 12-34, 2006

[50] J. G. Boulton, P. M. Allen, and C. Bowman, Embracing Complexity: Strategic Perspectives for an Age of Turbulence. Oxford, UK: Oxford University Press, 2015.

[51] S. Milgram, "The small-world problem," Psychology Today, vol. 1, no. 1, pp. 61-67, May 1967.

[52] M. E. J. Newman, Networks: An Introduction, 2nd ed. New York: Oxford University Press, 2018.

[53] A. Helmy, "Small worlds in wireless networks," IEEE Communications Letters, vol. 7, no. 10, pp. 490-492, Oct. 2003.

[54] V. Latora and M. Marchiori, "Efficient behavior of small-world networks," Phys. Rev. Lett., vol. 87, no. 19, Oct. 2001, Art. no. 198701.

[55] X. Liao, A. V. Vasilakos, and Y. He, "Small-world human brain networks: Perspectives and challenges," Neuroscience \& Biobehavioral Reviews, vol. 77, pp. 286-300, June 2017.

[56] G. J. Pottie, "System design choices in personal communications," IEEE Personal Communications, vol. 2, no. 5, pp. 50-67, Oct. 1995.

[57] J. G. Proakis, Digital Communications. New York: McGraw-Hill, 2001.

[58] U. Madhow, Fundamentals of Digital Communication. Cambridge, UK: Cambridge University Press, 2008.

[59] J. H. Holland, Hidden Order: How Adaptation Builds Complexity. Addison Wesley, 1995.

[60] H. Bossel, Systems and Models: Complexity, Dynamics, Evolution, Sustainability. Norderstedt, Germany: Books on Demand, 2007.

[61] Y. Wang, "Network management and orchestration using artificial intelligence: Overview of ETSI ENI," IEEE Communications Standards Magazine, vol. 2, no. 4, pp. 58-65, Dec. 2018.

[62] G. N. Saridis, "Toward the realization of intelligent controls," Proc. IEEE, vol. 67, no. 8, pp. 1115-1133, Aug. 1979.

[63] Y. S. Wu, “Constant capacity', DSP architecture - an historical prospective," in Underwater Acoustic Data Processing, Y. T. Chan, Ed. Dordrecht: The Netherlands: Kluwer Academic Publishers, 1989, pp. 609-627.

[64] J. Rexford et al., "Network-wide decision making: Toward a wafer-thin control plane," in Proc. HotNets, 2004, pp. 59-64.

[65] R. T. Marler and J. S. Aurora, "Survey of multiobjective optimization methods for engineering," Struct. Multidisciplinary Optim., vol. 26, no. 6, pp 369-395, Apr. 2004 